\title{
On the Alignment of Shapes Represented by Fourier Descriptors
}

\author{
Karl Sjöstrand ${ }^{1}$, Anders Ericsson ${ }^{2}$ and Rasmus Larsen ${ }^{1}$ \\ ${ }^{1}$ Informatics and Mathematical Modelling, Technical University of Denmark, Denmark \\ ${ }^{2}$ Centre for Mathematical Sciences, Lund Institute of Technology, Lund University, Sweden
}

\begin{abstract}
The representation of shapes by Fourier descriptors is a time-honored technique that has received relatively little attention lately. Nevertheless, it has its benefits and is suitable for describing a range of medical structures in two dimensions. Delineations in medical applications often consist of continuous outlines of structures, where no information of correspondence between samples exist. In this article, we discuss a Euclidean alignment method that works directly with the functional representation of Fourier descriptors, and that is optimal in a leastsquares sense. With corresponding starting points, the alignment of one shape onto another consists of a single expression. If the starting points are arbitrary, we present a simple algorithm to bring a set of shapes into correspondence.

Results are given for three different data sets; 62 outlines of the corpus callosum brain structure, 61 outlines of the brain ventricles, and 50 outlines of the right lung. The results show that even though starting points, translations, rotations and scales have been randomized, the alignment succeeds in all cases.

As an application of the proposed method, we show how high-quality shape models represented by common landmarks can be constructed in an automatic fashion. If the aligned Fourier descriptors are inverse transformed from the frequency domain to the spatial domain, a set of roughly aligned landmarks are obtained. The positions of these are then adjusted along the contour of the objects using the minimum description length criterion, producing ample correspondences. Results on this are also presented for all three data sets.
\end{abstract}

Keywords: Alignment, Shape

\section{INTRODUCTION}

This paper revisits the representation of shapes using Fourier descriptors and discusses a simple alignment method. Over the last decade, the use of Fourier descriptors for representing outlines of shapes has received relatively little attention. The method is burdened by a couple of well-known disadvantages. Since it uses complex numbers to represent $x$ and $y$-coordinates, it is limited to two dimensions, and since Fourier analysis is best suited for periodic functions, it is almost exclusively used for objects that can be decomposed into one or more closed curves. However, such curves are not uncommon and often arise from delineations of medical data; brain structures, lungs and the heart muscle are examples of suitable candidates. Further, Fourier descriptors have some appealing advantages. It is a functional representation which means that the shapes can be evaluated at any point along the outline. The interpretation of a shape as the superposition of a series of curves with increasing frequency provides a natural form of regularization. The low frequency curves provide the gross geometrical information while higher order curves add the details. A cutoff frequency may therefore be chosen such that a suitable tradeoff between approximation of input data and curve smoothness is achieved. Outlines may be represented using different numbers of input points across a data set, and Fourier descriptors can be calculated with high computational efficiency using the fast Fourier transform.

Further author information:

Karl Sjöstrand: E-mail: kas@imm.dtu.dk, Telephone: +45 45253423

Anders Ericsson: E-mail: anders.ericsson@math.lth.se, Telephone: +46 462220553

Rasmus Larsen: E-mail: rl@imm.dtu.dk, Telephone: +45 45253415 
The interpretation of shapes in the frequency domain dates back to a 1960 technical report by Cosgriff, ${ }^{1}$ however, it was Granlund ${ }^{2}$ who first interpreted simple closed curves as periodic complex functions and represented these using Fourier descriptors. Another important early paper is that of Zahn and Roskies ${ }^{3}{ }^{2}$ where an outline representation is used that is independent of similarity transformations. However, this method comes with a number of disadvantages and is not considered here. These early papers, as well as some later work, ${ }^{4}$ focused mainly on finding shape descriptors for use in shape classification and did not approach the issue of shape modelling. The generalization of Fourier descriptors to 3D is investigated in several papers, see e.g Ref. $5-7$.

This paper defines the alignment of one shape with respect to another as the Euclidean similarity transformation that translates, rotates and scales the shape according to some distance measure. This choice has been made for two reasons. First, it is an intuitive choice based on the definition of shape by Kendall ${ }^{8}$; second, it has been the choice of earlier papers describing the alignment of shapes represented by Fourier descriptors. ${ }^{9-12}$

Pavlidis $^{9}$ and Staib and Duncan ${ }^{10}$ describe similar methods for alignment of Fourier descriptors. Interpreting the Fourier series expansion as the superposition of a number of ellipses with increasing angular frequency, the alignment is based on modifying the shape such that the major axis of the first order ellipse of each shape has equal length and orientation. Neumann et al. ${ }^{11}$ and Folkers and Samet ${ }^{13}$ are more recent examples of this alignment scheme. Persoon and $\mathrm{Fu}^{12}$ perform least-squares optimal ordinary Procrustes analysis by direct optimization of rotation, scale and starting point.

As an application of the alignment method, we use Fourier descriptors as an intermediate representation for turning unaligned outlines devoid of correspondence information into shape models represented by landmarks. As part of this method, the minimum description length (MDL) framework is used. MDL has been shown to be an efficient method for refining correspondences along the outlines of a set of shapes, cf. Ref. 14 and Ref. 15.

This paper presents expressions for exact calculations of the area and the center of gravity of shapes described by Fourier descriptors. Persoon and $\mathrm{Fu}^{12}$ presents an expression for the area which is slightly different but equivalent to the one derived here. To our knowledge, the expression for center of gravity is previously unreported.

The data used in the experiments consists of 62 outlines of the mid-sagittal cross-section of the corpus callosum - the nerve fibre bundle connecting the hemispheres of the brain, 61 outlines of the lateral brain ventricles drawn from axial slices of volumetric MR data, and 50 outlines of the right lung collected from chest radiographs. The MR images, from which the corpus callosum and ventricle data is derived, stem from the LADIS (Leukoaraiosis and DISability) study, ${ }^{16}$ a pan-European study involving 12 hospitals and more than 600 patients.

\section{METHODS}

Fourier descriptors are, simply put, the coefficients of a Fourier transform of ordinary spatial data. This paper uses a combination of a discrete Fourier transform and a Fourier series ${ }^{17}$ to calculate the Fourier coefficients $c_{k}$ and to recover the continuous boundary curve $f(t)$ respectively,

$$
\begin{aligned}
& c_{k}=\frac{1}{n} \sum_{j=0}^{n-1} e^{-2 \pi i j k / n} f_{j}, \\
& f(t)=\sum_{k=-(N-1) / 2}^{(N-1) / 2} c_{k} e^{i k t} .
\end{aligned}
$$

Here $f_{j}=x_{j}+i y_{j}, j=0 \ldots n-1$, represents the set of input points ( $n$ is odd in this formulation), $N \leqslant n$ is the number of Fourier coefficients used in the reconstruction, and $i$ is the complex unit. Equation 1 can be rewritten in matrix form,

$$
\mathbf{c}=\mathbf{B f},
$$

where element $(k, j)$ of $\mathbf{B}$ is equal to $e^{-2 \pi i j k / n} / n$. It is well known that the Fourier basis is orthogonal, and it can be seen that $\mathbf{B}^{*} \mathbf{B}=\mathbf{I}_{n} / n$, where $\mathbf{B}^{*}$ is the complex conjugate transpose of $\mathbf{B}$ and $\mathbf{I}_{n}$ is the identity matrix of size $n$. This property helps in understanding the similarities between the treatment of Fourier coefficients and 
the spatial input; the Fourier coefficients are simply a rotation and a uniform scaling of the input data. Equation 2 may be rewritten in matrix form in a similar fashion,

$$
f(t)=\mathbf{h}(t)^{T} \mathbf{c},
$$

where $h_{k}(t)=e^{i k t}$. However, in the remainder of this paper, we will use the slightly different form

$$
g(t)=\mathbf{h}(t)^{*} \mathbf{c},
$$

which simplifies notation. The difference between the two is the direction of the curve, since $\mathbf{h}(t)^{*}=\mathbf{h}(-t)^{T}$ for this choice of basis function, that is, $g(t)=f(-t)$. We postpone a discussion on the impact of drawing directions until Section 2.1.

Another useful way of expressing Equation 2 is the form used in Ref. 10. $f(t)$ can be divided into separate functions for $x$ and $y$,

$$
\left[\begin{array}{l}
x(t) \\
y(t)
\end{array}\right]=\left[\begin{array}{l}
p_{0} \\
r_{0}
\end{array}\right]+\sum_{k=1}^{(N-1) / 2}\left[\begin{array}{ll}
p_{k} & q_{k} \\
r_{k} & s_{k}
\end{array}\right]\left[\begin{array}{l}
\cos k t \\
\sin k t
\end{array}\right]
$$

where $c_{k}+c_{-k}=p_{k}+i r_{k}, i\left(c_{k}-c_{-k}\right)=q_{k}+i s_{k}$ and $c_{0}=p_{0}+i r_{0}$. From Equation 6 , the well-known geometric interpretation of Fourier synthesis can be spotted. Each $k$ describes an ellipse with axes $p_{k}+i r_{k}$ and $q_{k}+i s_{k}$ and angular frequency $k$. This provides a natural form of shape regularization; low frequency ellipses account for the major shape features while higher frequencies add the details. $N \leqslant n$ may therefore be chosen to achieve a specific level of detail.

\subsection{Alignment}

Larsen ${ }^{18}$ provides a general treatment of functional 2D Procrustes analysis. This section investigates the implications of the results for the specific functional representation of Fourier descriptors. To clarify the explanation, the main results from Ref. 18 will be recapitulated here.

The centroid $\bar{f}$ of a functional curve is defined as the mean position of a particle travelling once around a closed curve, $\bar{f}=\frac{1}{l} \int_{0}^{l} f(s) d s$. Shapes with $\bar{f}=0$ are said to be centered. Consider two centered curves of arbitrary functional representation, $y(t), w(t):[0, l] \rightarrow \mathbb{C}$. The full functional Procrustes fit of $w$ onto $y$ is

$$
w^{P}(t)=\hat{a}+\hat{b} w(t)
$$

where $(\hat{a}, \hat{b}) \in \mathbb{C}$ are chosen to minimize

$$
\int_{0}^{l}\|y(s)-b w(s)-a\|^{2} d s .
$$

This expression can be solved for $a$ and $b$ by differentiating and setting to zero. The minimizing parameters $\operatorname{are}^{18}$

$$
\hat{a}=0, \quad \hat{b}=\frac{\int_{0}^{l} w(s)^{*} y(s) d s}{\int_{0}^{l} w(s)^{*} w(s) d s} .
$$

What do these results imply for Fourier descriptors? To investigate this, the boundary representation of Equation 5 is inserted. The expression for the centroid becomes

$$
\bar{f}=\frac{1}{2 \pi} \int_{0}^{2 \pi} \mathbf{h}(s)^{*} \mathbf{c} d s=\frac{1}{2 \pi} \int_{0}^{2 \pi} \mathbf{h}(s)^{*} d s \mathbf{c} .
$$

The integral is calculated for one or more complete periods of pure sine and cosine functions, which therefore integrate to zero. The exception is $k=0$, for which the basis function is 1 . The expression therefore simplifies to

$$
\bar{f}=c_{0} .
$$


This shows that letting $c_{0}$ denote the overall translation of a shape, as is done in earlier work, ${ }^{1-3,9-12}$ is indeed a sensible choice. In other words, the centering of a shape amounts to setting $c_{0}=0$. The least-squares optimal transformation $\hat{b}$ that rotates and scales a shape $w(t)=\mathbf{h}(t)^{*} \mathbf{w}$ with respect to another $y(t)=\mathbf{h}(t)^{*} \mathbf{y}$ can be simplified in a similar manner by insertion of (5) into (9),

$$
\hat{b}=\frac{\int_{0}^{2 \pi}\left(\mathbf{h}(s)^{*} \mathbf{w}\right)^{*}\left(\mathbf{h}(s)^{*} \mathbf{y}\right) d s}{\int_{0}^{2 \pi}\left(\mathbf{h}(s)^{*} \mathbf{w}\right)^{*}\left(\mathbf{h}(s)^{*} \mathbf{w}\right) d s}=\frac{\mathbf{w}^{*} \int_{0}^{2 \pi} \mathbf{h}(s) \mathbf{h}(s)^{*} d s \mathbf{y}}{\mathbf{w}^{*} \int_{0}^{2 \pi} \mathbf{h}(s) \mathbf{h}(s)^{*} d s \mathbf{w}}=\frac{\mathbf{w}^{*} \mathbf{y}}{\mathbf{w}^{*} \mathbf{w}},
$$

where $\mathbf{w}$ and $\mathbf{y}$ are the Fourier coefficients of shape $w(t)$ and $y(t)$ respectively. The integrals $\int_{0}^{2 \pi} \mathbf{h}(s) \mathbf{h}(s)^{*} d s$ simplify to $2 \pi \mathbf{I}_{N}$ since $\mathbf{h}(s)$ is an orthogonal basis set. The result resembles the familiar equation for ordinary Procrustes analysis of landmarks in two dimensions. ${ }^{19}$ Note, however, that the assumption of centered shapes translates to $\sum_{k} w_{k}=0$ for landmarks and $w_{0}=0$ for Fourier descriptors.

The alignment of one shape with respect to another is normally denoted ordinary Procrustes analysis, while the alignment of two or more shapes with respect to their common mean is known as general Procrustes analysis. Assuming the shapes are already aligned, the sample mean shape is found by,

$$
\bar{f}(t)=\frac{1}{m} \sum_{i=1}^{m} f_{i}(t)=\frac{1}{m} \sum_{i=1}^{m} \mathbf{h}(t)^{*} \mathbf{c}_{i}=\mathbf{h}(t)^{*} \overline{\mathbf{c}} .
$$

where $m$ is the number of shapes in the training set and $\mathbf{c}_{i}$ are the Fourier coefficients of the $i$ th shape. That is, the coefficients of the sample mean shape are merely the sample mean vector of coefficients. However, the mean shape cannot be calculated in this fashion unless the shapes are aligned. Larsen ${ }^{18}$ provides a second useful result.

The coefficients of the full functional Procrustes estimate of the mean curve of the centered (but not necessarily aligned) sample of curves $f_{i}(t)$ are $\overline{\mathbf{c}}=\mathbf{L}^{-1} \mathbf{v}$, where $\mathbf{v}$ is the eigenvector corresponding to the largest eigenvalue of the Hermitian complex sum of squares and products matrix,

$$
\mathbf{C}=\sum_{i=1}^{m} \frac{\left(\mathbf{L} \mathbf{c}_{i}\right)\left(\mathbf{L} \mathbf{c}_{i}\right)^{*}}{\left(\mathbf{L} \mathbf{c}_{i}\right)^{*}\left(\mathbf{L} \mathbf{c}_{i}\right)}
$$

where $\mathbf{L}^{*} \mathbf{L}=\int_{0}^{2 \pi} \mathbf{h}(s) \mathbf{h}(s)^{*} d s$.

Following the discussion earlier, $\mathbf{L}=\sqrt{2 \pi} \mathbf{I}_{N}$ for Fourier descriptors, and the expression simplifies into

$$
\mathbf{C}=\sum_{i=1}^{m}\left(\mathbf{c}_{i}^{*} \mathbf{c}_{i}\right)^{-1} \mathbf{c}_{i} \mathbf{c}_{i}^{*}
$$

The estimated sample mean is unique up to an arbitrary rotation and the scaling is fixed since $\|\mathbf{v}\|=1$. This means in practice that setting $\overline{\mathbf{c}}=\mathbf{v}$ is a perfectly good choice. What remains of the general Procrustes analysis is to align all shapes with respect to $\bar{f}(t)=\mathbf{h}(t)^{*} \overline{\mathbf{c}}$ using Equation 11 .

There are a number of assumptions on the input data in the discussion on alignment above. The spatial input, gathered in the complex vectors $\mathbf{f}_{i}, i=1 \ldots m$, must be ordered along the boundaries of the shapes. One may think of the elements of $\mathbf{f}_{i}$ as samples of the continuous function $f_{i}(t)$ that is to be estimated. Moreover, the curve direction must be the same for all shapes. The drawing direction of a simple closed curve is defined as counter clock-wise if the region defined by the curve is to the left of curve. The area calculations presented in Section 2.2 assumes that the curve is drawn counter clock-wise. If the curve is drawn clock-wise the area will be negative. A suitable measure of drawing direction is therefore the sign of the area. If the drawing direction of a shape needs be switched, it can be performed by setting $c_{k}=c_{-k}$ since

$$
f(-t)=\sum_{k=-(N-1) / 2}^{(N-1) / 2} c_{k} e^{-i k t}=\sum_{k=-(N-1) / 2}^{(N-1) / 2} c_{-k} e^{i k t} .
$$


A more involved assumption is that the parameter $t$ in Equation 2 corresponds across samples. That is, any $t \in[0,2 \pi)$ should ideally correspond to the same geometrical/anatomical position on all shapes. This is true if the starting points on all shapes correspond, and the speed functions $s(t)=\left|f^{\prime}(t)\right|$ along the boundaries are the same. We will present a simple algorithm for finding common starting points, and provide some empirical evidence that the speed functions are sufficiently similar to provide ample correspondences.

Let the starting point position parameter be denoted by $\delta$. The boundary function becomes

$$
f(t+\delta)=\mathbf{h}(t+\delta)^{*} \mathbf{c}=[\Lambda \mathbf{h}(t)]^{*} \mathbf{c}=\mathbf{h}(t)^{*} \Lambda^{*} \mathbf{c},
$$

where $\Lambda$ is a diagonal matrix with $\Lambda_{k k}=e^{i k \delta}$. The addition of an arbitrary starting point turns the expression for the alignment of $w(t+\delta)$ onto $y(t)$ (cf. Equation 11) into

$$
\hat{b}=\frac{\mathbf{w}^{*} \Lambda \mathbf{y}}{\mathbf{w}^{*} \mathbf{w}} .
$$

This expression is dependent on the starting position $\delta$, which is unknown, so $\hat{b}$ cannot be calculated directly. One therefore has to resort to numerical optimization of some sort. One possibility is to perform an ordinary Procrustes analysis by simultaneous optimization of $b$ and $\delta$ as in Ref. 12. However, to align a complete set of shapes to their mutual mean becomes impractical and, more importantly, the parameter space will contain a number of local minima. Instead, Equation 16 may be used to estimate $\hat{b}$ for a given starting point, reducing the problem to one of numerically estimating the optimal $\delta$. The cost function to be minimized is

$$
q(\delta)=\left\|\mathbf{y}-\frac{\mathbf{w}^{*} \Lambda \mathbf{y}}{\mathbf{w}^{*} \mathbf{w}} \Lambda^{*} \mathbf{w}\right\|^{2} .
$$

Again, the basis functions cancel out and are excluded. Intuitively, this function is minimal when the starting points correspond. Objects that are roughly elliptical, such as the corpus callosum brain structure, will have two local minima, one for corresponding starting points, and one for a misalignment of approximately $\pi$ radians, when the object is flipped along its long axis. Star-shaped objects, such as the ventricle data set, will have one local minimum every time the misalignment puts two extruding parts of the shapes on top of each other. The conclusion is that for objects that are not overly complex, the cost function is smooth and contains a low number of local minima. The optimum can therefore be found using a range of available methods for global optimization. Here, a simple method is used, where the fit function of Equation 17 is sampled at regular intervals in the range $[0,2 \pi)$. The number of samples is a tunable parameter that reflects the complexity of the present object class. The value of $\delta$ giving the lowest cost $q(\delta)$ is chosen as a starting point for a standard gradient decent optimization algorithm, fine-tuning the estimated optimal $\delta$.

The entire algorithm for aligning a set of digitized outlines with no information on correspondence is given in Algorithm 1.

\subsection{Area and Center of Gravity}

The functional nature of Fourier descriptors can be used to extract shape features. Presented here are expressions for area and center of gravity that turn out to be functions of the descriptors only. These quantities are therefore easy to calculate and give precise results. Both expressions spawn from Green's theorem, which provides a useful relationship between a boundary function $\partial D$, such as those described by Fourier descriptors, and the region $D$ defined by the boundary. For completeness, the theorem is recapitulated here.

Let $f(x, y)$ and $g(x, y)$ be differentiable functions defined on an open set $\Omega$ in two dimensions. If the compact subset $D \subset \Omega$ has a boundary $\partial D$, then

$$
\int_{\partial D} f(x, y) d x+g(x, y) d y=\iint_{D}\left(\frac{\partial g(x, y)}{\partial x}-\frac{\partial f(x, y)}{\partial y}\right) d x d y .
$$

First, the theorem is used to derive an expression for the area of a shape. Assume a small area element of the region $D$ has width $d x$, height $d y$ and area $d x d y$. The total area of the region can then be written $A=\iint_{D} d x d y$. 


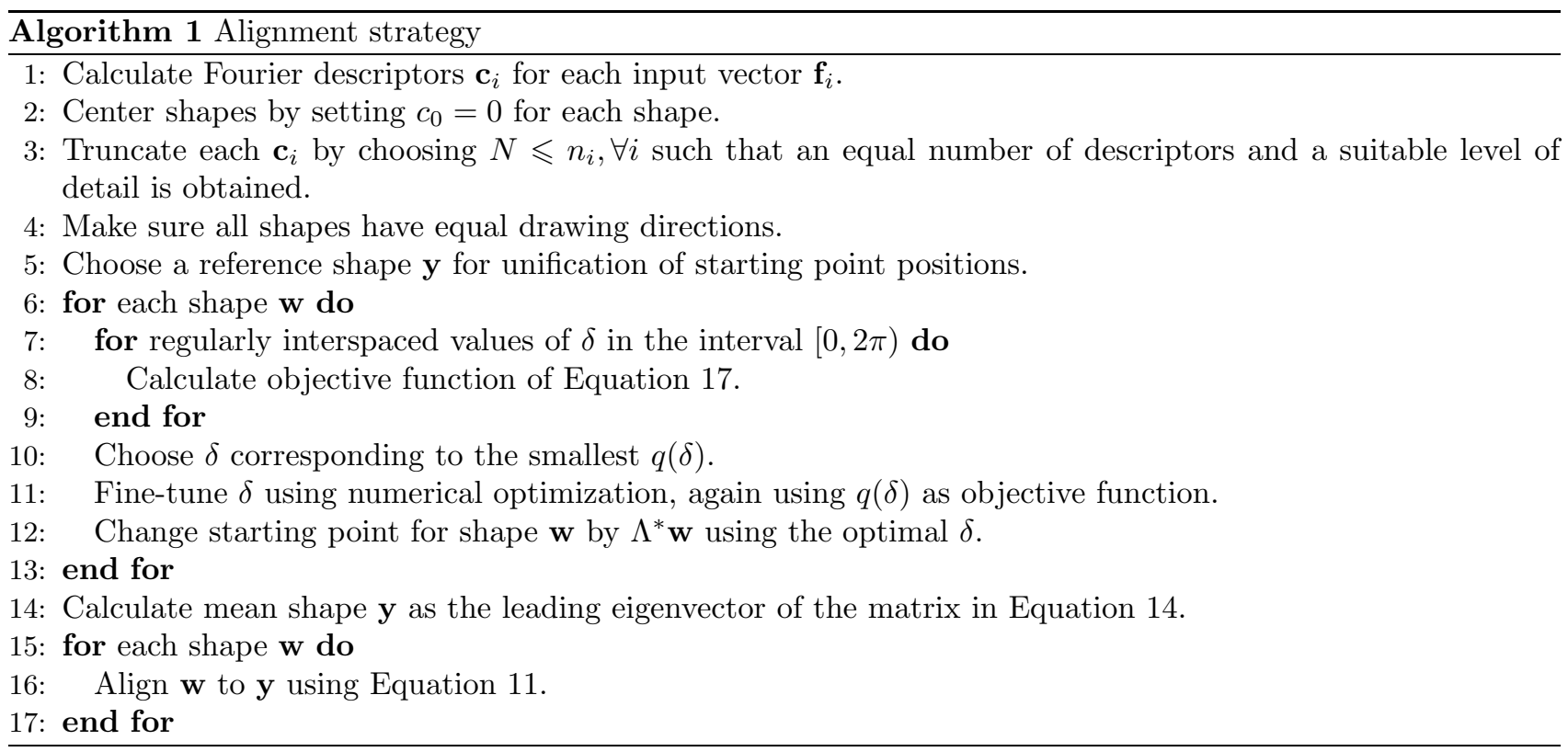

Using Green's theorem this can be reformulated using a line integral,

$$
A=\iint_{D} d x d y=\frac{1}{2} \int_{\partial D} x d y-y d x=\frac{1}{2} \int_{\partial D} x(t) y^{\prime}(t)-y(t) x^{\prime}(t) d t .
$$

Inserting the expressions for $x(t)$ and $y(t)$ from Equation 6, along with their derivatives, this results in a general expression for the area of shapes represented by Fourier descriptors. This expression can be greatly reduced, mainly since the integral is calculated over one or more periods of sine and cosine functions, which equal zero. The remaining terms are those including cosine functions with zero frequency. This yields

$$
A=\pi \sum_{k=1}^{(N-1) / 2} k\left|\begin{array}{ll}
p_{k} & q_{k} \\
r_{k} & s_{k}
\end{array}\right|=\pi \sum_{k=-(N-1) / 2}^{(N-1) / 2} k\left|c_{k}\right|^{2} .
$$

The latter form of the expression is due to Persoon and Fu. ${ }^{12}$

Green's theorem also makes it possible to derive an expression for the center of gravity (CoG) of a given shape. The CoG is the point on a surface where the total moment in any direction equals zero. As before, $d x$ and $d y$ are the width and height of a small area unit. The mass of this unit at position $(x, y)$ is $d x d y \delta(x, y)$ where $\delta(x, y)$ is the density function of the material. Let $x_{\operatorname{cog}}$ and $y_{\operatorname{cog}}$ be the $x$ and $y$ coordinates of the CoG to be found. The area element $d x d y$ introduces a moment in the $x$ and $y$ directions with respect to $x_{\operatorname{cog}}, y_{\operatorname{cog}}$ which is $\delta(x, y)\left(x-x_{\operatorname{cog}}, y-y_{\operatorname{cog}}\right) d x d y$. Summing up the moments for the whole surface and equating to zero gives

$$
\begin{gathered}
\iint_{D} \delta(x, y)\left(x-x_{\operatorname{cog}}, y-y_{\operatorname{cog}}\right) d x d y=(0,0) \Leftrightarrow\left(x_{\operatorname{cog}}, y_{\operatorname{cog}}\right) \iint_{D} \delta(x, y) d x d y=\iint_{D} \delta(x, y)(x, y) d x d y \Leftrightarrow \\
\left(x_{\operatorname{cog}}, y_{\operatorname{cog}}\right)=\frac{1}{M} \iint_{D} \delta(x, y)(x, y) d x d y \Leftrightarrow\left(x_{\operatorname{cog}}, y_{\operatorname{cog}}\right)=\frac{1}{M} \int_{\partial D} \delta(x, y)(-x y d x, x y d y),
\end{gathered}
$$

where $M$ is the total mass of the shape. Assuming the density is uniform over the whole shape, this simplifies to

$$
\left(x_{\operatorname{cog}}, y_{\operatorname{cog}}\right)=\frac{1}{A} \int_{\partial D}(-x y d x, x y d y)=\frac{1}{A} \int_{\partial D}\left(-x(t) y(t) x^{\prime}(t), x(t) y(t) y^{\prime}(t)\right) d t,
$$


where $A$ is the area of the shape. Again, the expressions for $x(t)$ and $y(t)$ from Equation 6 are used. After some simplification, the expressions reduce to functions of the Fourier coefficients only.

$$
\begin{gathered}
x_{c o g}=\frac{\pi}{2 A} \sum_{a=2}^{n} \sum_{b=1}^{a-1} a\left(q_{b} s_{a-b} q_{a}+p_{b} s_{a-b} p_{a}+q_{b} r_{a-b} p_{a}-p_{b} r_{a-b} q_{a}\right)+ \\
b\left(-q_{a} s_{a-b} q_{b}-q_{a-b} s_{a} q_{b}-p_{a} s_{a-b} p_{b}+p_{a-b} s_{a} p_{b}+q_{a} r_{a-b} p_{b}-q_{a-b} r_{a} p_{b}-p_{a} r_{a-b} q_{b}-p_{a-b} r_{a} q_{b}\right) \\
y_{c o g}=\frac{\pi}{2 A} \sum_{a=2}^{n} \sum_{b=1}^{a-1} a\left(p_{b} r_{a-b} s_{a}-q_{b} s_{a-b} s_{a}-p_{b} s_{a-b} r_{a}-q_{b} r_{a-b} r_{a}\right)+ \\
b\left(p_{a} r_{a-b} s_{b}+p_{a-b} r_{a} s_{b}+q_{a} s_{a-b} s_{b}+q_{a-b} s_{a} s_{b}+p_{a} s_{a-b} r_{b}-p_{a-b} s_{a} r_{b}-q_{a} r_{a-b} r_{b}+q_{a-b} r_{a} r_{b}\right)
\end{gathered}
$$

While these expressions are not as simple as the expression for the area above, they are straight-forward to implement.

\subsection{Application: Optimal Shape Models from Outlines}

The correspondences attained using Algorithm 1 are approximate, but suffice for most applications. However, shapes described by landmarks may be preferred since they are a more common choice of representation. Fourier descriptors may work as an intermediate step in this application, providing a suitable domain to solve the registration task. Following the alignment described above, we propose to rotate the Fourier descriptors back into the spatial (landmark) domain using the inverse Fourier transform,

$$
\tilde{f}_{j}=\sum_{j=0}^{N-1} e^{2 \pi i j k / N} c_{k} \Leftrightarrow \tilde{\mathbf{f}}=\mathbf{B}^{-1} \mathbf{c}=N \mathbf{B}^{*} \mathbf{c} .
$$

Note that $\tilde{\mathbf{f}}(N \times 1)$ denotes a vector of aligned landmarks while $\mathbf{f}(n \times 1)$ from Equation 3 represents their unaligned counterpart. The aligned shapes are described by an equal amount of Fourier descriptors, corresponding to an equal amount of landmarks spread out equidistantly along the boundaries of the shapes in terms of the parameter $t$. These are the landmarks attained using the inverse transformation which, assuming the speed functions are similar (see Section 3), will be in approximate correspondence. To improve these correspondences, we propose to adjust the landmark positions such that the description length of the resulting model is minimized.

The description length (DL) provides a way of evaluating a shape model. The cost in description length is derived from information theory and corresponds to the effort that is needed to send the model and the shapes bit by bit. The minimum description length (MDL) principle searches iteratively for a model that transmits the data the cheapest. The cost function makes a tradeoff between a model that is general (can represent any instance of the object), specific (it can only represent valid instances of the object) and compact (it can represent the variation with as few parameters as possible). Davies and Cootes ${ }^{20}$ relate these ideas to the principle of Occam's razor: the simplest explanation generalizes the best.

Since the idea of using MDL for shape models was first published, ${ }^{20}$ the cost function has been refined and tuned. Here, an improved version of the cost function, stated in Ref. 21 and derived in Ref. 22, is used.

$$
D L=\sum_{\lambda_{i} \geqslant c}\left(1+\log \frac{\lambda_{i}}{\lambda_{c}}\right)+\sum_{\lambda_{i}<\lambda_{c}, \lambda_{i} \geqslant \lambda_{t}} \frac{\lambda_{i}}{\lambda_{c}} .
$$

The scalars $\lambda_{i}$ are the eigenvalues of the covariance matrix $\mathbf{X}^{T} \mathbf{X} / N$, where $\mathbf{x}_{i}=\left[\operatorname{Re}\left(\tilde{\mathbf{f}}_{i}\right)^{T} \operatorname{Im}\left(\tilde{\mathbf{f}}_{i}\right)^{T}\right]^{T}$. The constants $\lambda_{c}$ and $\lambda_{t}$ are calculated from $\Delta$, which describes the precision of the landmark coordinates. Information can only be sent up to a certain degree of accuracy and $\Delta$ expresses this threshold. The constant $\lambda_{c}=2 \Delta$ is the limit between what is expected to be information and what is expected to be noise. When the range of the data is smaller than $\Delta$ no information needs to be sent. This limit is set by $\lambda_{t}$. There are two important properties of this cost function. It is more intuitive than those formerly presented and the derivative is continuous.

To improve correspondences, we allow the landmarks to move along the boundary of each shape, such that the cost function in Equation 23 is minimized. The algorithm to convert non-corresponding outlines into description length optimal landmark representations is summarized in Algorithm 2. 


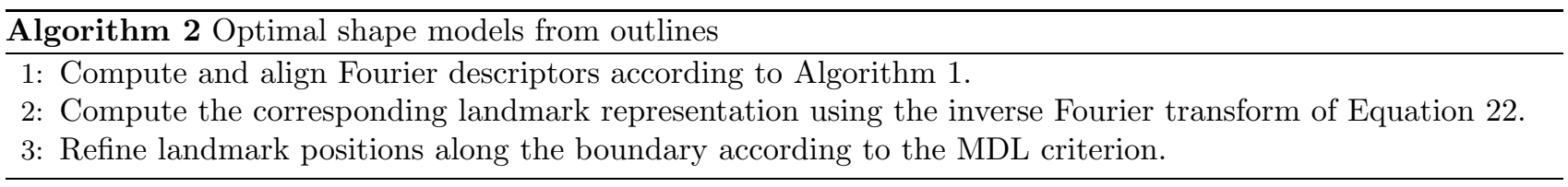

\section{RESULTS}

Figure 1 shows alignment results for all three data sets. Before alignment, translation has been filtered out and remaining parameters - scale, orientation and starting points - are randomized. The smallest number of samples along the boundaries necessary to provide a suitable starting point for each shape in the numerical optimization step (cf. steps 7-10 in Algorithm 1) was 7, 13, and 15 for the corpus callosum, lungs and ventricles data sets respectively. This is according to expectation since the numbers reflect the complexity of the objects.
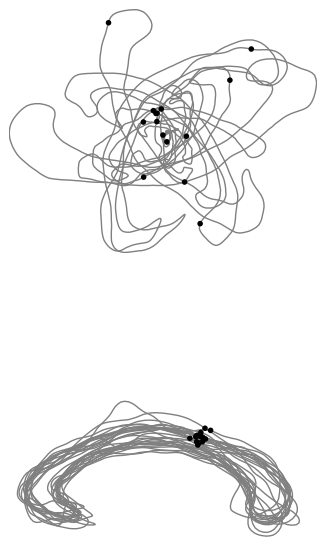

(a) Corpus callosum
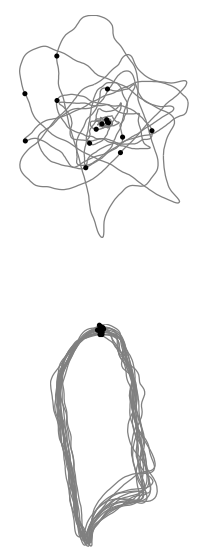

(b) Lungs
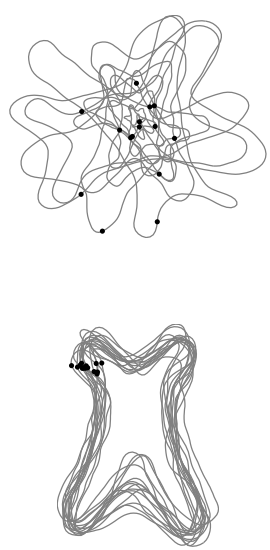

(c) Ventricles

Figure 1. Alignment of the corpus callosum, lung and ventricle data sets. The top row shows the shapes with randomized starting points, orientation and scale, and the bottom row shows the corresponding aligned shapes. To clarify the results, only a few samples of each data set are shown. Black dots denote starting point positions.

The alignment algorithm assumes that the cost function defined in Equation 17 is smooth and contains a low number of local minima. Figure 2 provides empirical evidence of the validity of this assumption. Each shape is intentionally misaligned onto the mean shape in terms of starting points by values in the interval $\delta \in[0,2 \pi)$. For each $\delta, q(\delta)$ is calculated, representing the sum of squared differences between the aligned shape and the mean shape. Starting points correspond at zero radians.

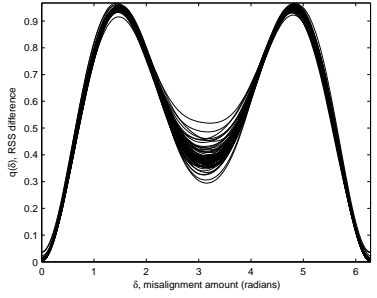

(a) Corpus callosum

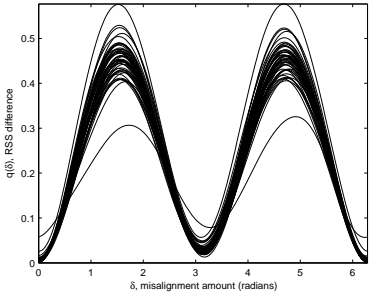

(b) Lungs

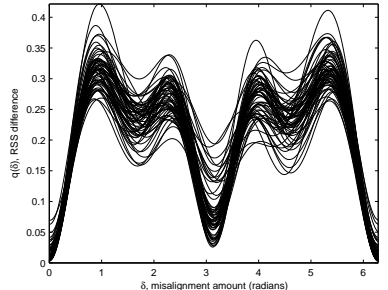

(c) Ventricles

Figure 2. The function $q(\delta)$ defined in Equation 17 in the interval $\delta \in[0,2 \pi)$. Alignment is calculated with respect to the mean shape. Each function has several minima, but the curves are smooth and the global minima are easily found.

The aligned Fourier descriptors may be viewed as an equal amount of landmarks placed at regular intervals along the boundary with respect to $t$ in Equation 2. Across a set of aligned shapes, these landmarks, or 
analogously, Fourier descriptors, will be in exact correspondence if the speed function $s(t)=\left|f^{\prime}(t)\right|$ is equal for all shapes. Figure 3 shows speed functions for 10 shapes from each data set. The data is normalized such that the mean speed function is equal to the line $y=x$. The deviation from the mean speed is then plotted along this line for each of the ten shapes. As can be seen, the deviations are relatively small, meaning that the Fourier descriptors are in relatively close correspondence.

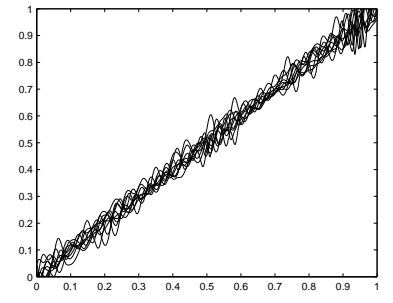

(a) Corpus callosum

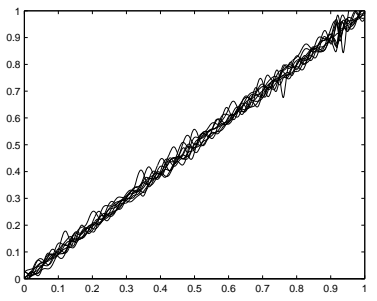

(b) Lungs

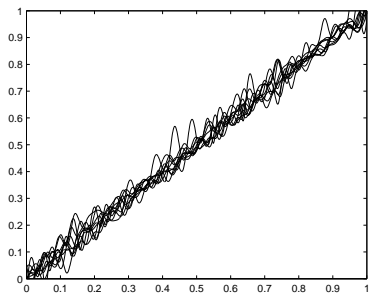

(c) Ventricles

Figure 3. The graph shows speed functions for the corpus callosum data set as offsets from the mean speed, represented by the line $y=x$. The limited deviations implies that the correspondences are relatively accurate.

Figure 4 shows the center of gravity and the centroid for a synthetic example and a shape from the corpus callosum data set. Alongside measurements of area, the center of gravity is a useful shape feature that may be beneficial in studies of morphology.
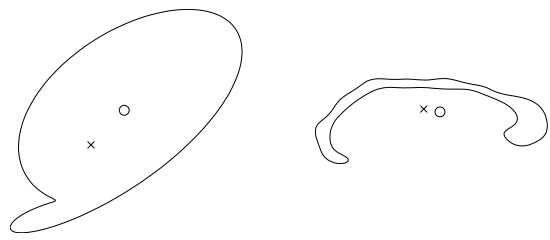

Figure 4. Center of gravity (o) versus the centroid ( $\mathrm{x}$ ) for a synthetic shape (left) and an example from the corpus callosum data set (right). As is evident, the center of gravity may differ significantly from the centroid. Such differences are due to variations in speed along the boundary. The synthetic shape has low curve speeds in the bottom left corner, and higher speeds in opposite corner. Since the centroid is the mean position of a particle travelling around the boundary, it is drawn towards areas of lower speeds and higher boundary complexity.

The automatic construction of description length optimal shape models is a minor but potentially useful application of Fourier descriptors. Figure 5 shows the landmark distributions for the ventricle data set before and after MDL refinement. Two important properties can be seen from these images. First, the correspondences provided by the Fourier descriptors alone are reasonably close to their refined counterparts. Hence they create adequate models for many applications. However, it is also seen that after MDL refinement, the distributions are smaller and/or more elongated. This intuitively leads to an increase in both specificity and compactness of the model. Figure 6 shows the generalization ability before and after MDL refinement for the lungs and ventricle data set. The results show that the refined model has markedly better generalization ability. These results motivate the use of MDL refinement if demands on model quality are high.

\section{DISCUSSION}

The use of Fourier descriptors for the representation of shape is not the most common choice for several reasons. It works best for closed contours, it does not generalize directly to 3D and it is best suited for objects without sharp corners. Perhaps adding to this list, is the complexity of previously reported alignment strategies. While this paper does not address the three first shortcomings, we present an alignment strategy which is more in line with alignment of shapes represented by landmarks. Another complication of Fourier descriptors is the lack 


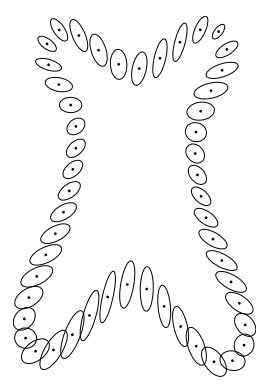

(a) Pre MDL refinement

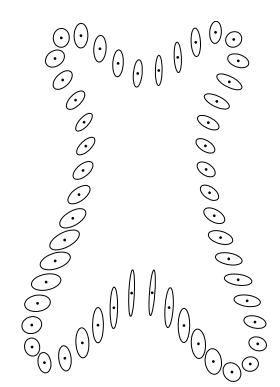

(b) Post MDL refinement

Figure 5. Landmark distributions for the ventricle data set before and after MDL refinement. Ellipses show one standard deviation. The results are similar, but the refined model has slightly lower variation, and the variation is more orthogonal with respect to the boundary.

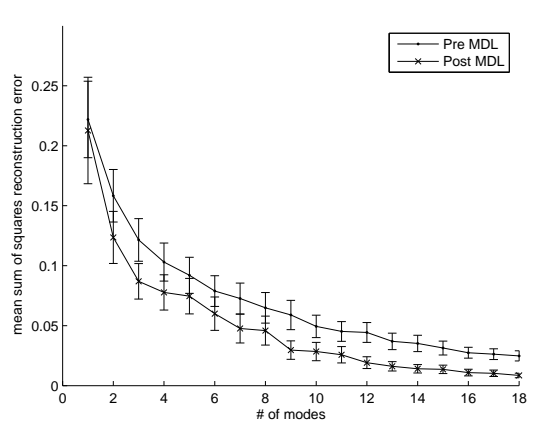

(a) Lungs

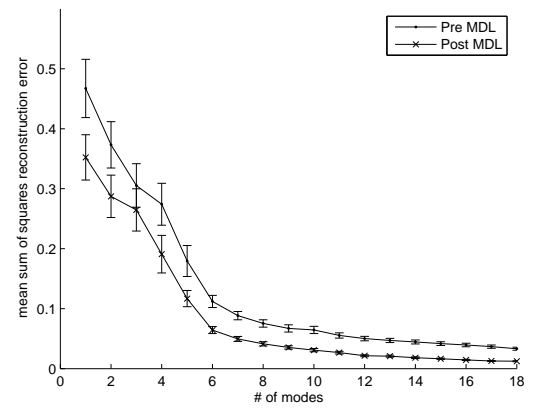

(b) Ventricles

Figure 6. Generalization ability as a function of number of modes of variation for the automatically generated landmarkbased models of lungs and ventricles. Generalization ability is measured as the reconstruction error in a leave-one-out study. After MDL optimization, the reconstruction error is significantly lower for both data sets.

of a principled way of choosing the amount of regularization, that is, picking an appropriate $N$ in Equation 2 . However, regularization is often called for, especially when working with medical data which may be sampled on coarse grids. The regularization property of Fourier descriptors may therefore be of great value.

The mathematical validity of the results in this paper are easily verified. However, they are built on assumptions that are difficult to make general statements about. The main assumption is that of correspondence across samples in terms of the variable $t$ in Equation 2. The results section provides empirical evidence that this assumption is valid for the present data sets. However, these results do not generalize to any class of objects.

Another assumption, related to the discussion above, is that the input points $f_{j}$ in Equation 1 are distributed similarly across samples. Several points within close distance of each other will reduce the speed in that area, while more spread out points leads to increased speed. As discussed, the speed functions must be roughly similar which explains the assumption. For objects that are represented by digitized outlines, this is usually not an issue, but e.g. missing data could cause a decrease in model quality.

Apart from the presentation and discussion on alignment, this paper presents two expressions for the exact calculation of area and center of gravity. We have also shown how the Fourier domain can be used to convert non-corresponding outlines into into a model represented by corresponding landmarks.

\section{ACKNOWLEDGMENTS}

Charlotte Ryberg and Egill Rostrup, The Danish Research Centre for Magnetic Resonance, Copenhagen University Hospital, Hvidovre, kindly provided MRIs used for corpus callosum and ventricle annotation. Dr. Bram 
van Ginneken, Image Sciences Institute, University Medical Center Utrecht, provided the chest radiographs for lung annotation. K. Sjöstrand was supported by The Technical University of Denmark, DTU.

\section{REFERENCES}

1. R. L. Cosgriff, "Identification of shape," tech. rep., Ohio State Univ. Res. Foundation, Columbus, OH, Dec. 1960.

2. G. H. Granlund, "Fourier preprocessing for hand print character recognition," IEEE Transactions on Computers C-21(2), pp. 195-201, 1972.

3. C. T. Zahn and R. Z. L. Roskies, "Fourier descriptors for plane closed curves," IEEE Transactions on Computers C-21(3), pp. 269-81, 1972.

4. P. Fieguth, P. Bloore, and A. Domsa, "Phase-based methods for Fourier shape matching," Acoustics, Speech, and Signal Processing, 2004. Proceedings. (ICASSP '04). IEEE International Conference on 3, pp. iii-425-8 vol.3, 2004.

5. L. H. Staib and J. S. Duncan, "Deformable Fourier models for surface finding in 3-D images," Proceedings of SPIE - The International Society for Optical Engineering 1808, pp. 90-104, 1992.

6. M. F. Wu and H. T. Sheu, "Representation of 3D surfaces by two-variable Fourier descriptors," Pattern Analysis and Machine Intelligence, IEEE Transactions on 20(8), pp. 858-863, 1998.

7. H. Zhang and E. Fiume, "Shape matching of 3-D contours using normalized Fourier descriptors," Shape Modeling International, 2002. Proceedings, pp. 261-268, 2002.

8. D. G. Kendall, "The diffusion of shape," Advances in Applied Probability 9, pp. 428-430, 1977.

9. T. Pavlidis, "Structural pattern recognition," Springer-Verlag, 1977.

10. L. H. Staib and J. S. Duncan, "Boundary finding with parametrically deformable models," Pattern Analysis and Machine Intelligence, IEEE Transactions on 14(11), pp. 1061 -1075, 1992.

11. A. Neumann, "Statistical shape model based segmentation of medical images," Computerized Medical Imaging and Graphics 22(2), pp. 133-143, 1998.

12. E. Persoon and K. S. Fu, "Shape discrimination using Fourier descriptors," IEEE Transactions on Pattern Analysis and Machine Intelligence PAMI-8(3), pp. 388-97, 1986.

13. A. Folkers and H. Samet, "Content-based image retrieval using Fourier descriptors on a logo database," Pattern Recognition, 2002. Proceedings. 16th International Conference on 3, pp. 521-524 vol.3, 2002.

14. R. H. Davies, T. F. Cootes, and C. J. Taylor, "A minimum description length approach to statistical shape modelling," Information Processing in Medical Imaging. 17th International Conference, IPMI 2001. Proceedings (Lecture Notes in Computer Science Vol.2082), pp. 50-63, 2001.

15. R. H. Davies, C. J. Twining, T. F. Cootes, J. C. Waterton, and C. J. Taylor, "A minimum description length approach to statistical shape modeling," Medical Imaging, IEEE Transactions on 21(5), pp. 525-537, 2002.

16. L. Pantoni, A. M. Basile, G. Pracucci, K. Asplund, J. Bogousslavsky, H. Chabriat, T. Erkinjuntti, F. Fazekas, J. M. Ferro, M. Hennerici, J. O'brien, P. Scheltens, M. C. Visser, L. O. Wahlund, G. Waldemar, A. Wallin, and D. Inzitari, "Impact of age-related cerebral white matter changes on the transition to disability - the LADIS study: Rationale, design and methodology," Neuroepidemiology 24(1-2), pp. 51-62, 2005.

17. W. L. Briggs and V. E. Henson, The DFT: An Owner's Manual for the Discrete Fourier Transform, SIAM, 1995.

18. R. Larsen, "Functional 2D Procrustes shape analysis," in 14th Scandinavian Conference on Image Analysis, LNCS, Springer Verlag, (Berlin Heidelberg), jun 2005.

19. I. L. Dryden and K. V. Mardia, Statistical Shape Analysis, John Wiley \& Sons, 1999.

20. R. H. Davies, T. F. Cootes, J. C. Waterton, and C. J. Taylor, "An efficient method for constructing optimal statistical shape models," in Medical Image Computing and Computer Assisted Intervention, pp. 57-65, 2001.

21. H. H. Thodberg, "Minimum description length shape and appearance models," in Proceedings of Information Processing in Medical Imaging (IPMI 2003), C. Taylor and J. A. Noble, eds., Lecture Notes In Computer Science (LNCS) 2732, Springer, 2003.

22. A. Ericsson and K. Åström, "Minimizing the description length using steepest descent," in Proc. British Machine Vision Conference, Norwich, United Kingdom, 2, pp. 93-102, 2003. 